\title{
Correction to: Morphology of the sulcus of the caudate process (Rouviere's sulcus) in a Greek population and a systematic review with meta-analysis
}

\author{
Dimitrios K. Manatakis ${ }^{1}$ (1) - Nikolaos Tasis ${ }^{1} \cdot$ Maria loanna Antonopoulou ${ }^{1} \cdot$ Christos Agalianos $^{1} \cdot$ Maria Piagkou $^{2}$. \\ John Tsiaoussis ${ }^{3} \cdot$ Konstantinos Natsis ${ }^{4}$. Dimitrios P. Korkolis ${ }^{5}$
}

Published online: 17 October 2021

(c) Japanese Association of Anatomists 2021

\section{Correction to: Anatomical Science International https://doi.org/10.1007/s12565-021-00628-5}

In the original publication of the article, fifth sentence under the "Abstract" heading should read as "The meta-analysis included 27 surgical and 11 cadaveric studies with 6761 cases in total." and the third sentence in the second paragraph under the "Results" heading should read as "A total of 38 studies ( $n=6761$ patients) ...".

Publisher's Note Springer Nature remains neutral with regard to jurisdictional claims in published maps and institutional affiliations.

The original article can be found online at https://doi.org/10.1007/ s12565-021-00628-5.

Dimitrios K. Manatakis

dmanatak@yahoo.gr; medp2011862@med.uoc.gr;

d.manatakis@hellenicnavy.gr

1 Department of Surgery, Athens Naval and Veterans Hospital, Deinokratous 70, 11521 Athens, Greece

2 Department of Anatomy, Faculty of Health Sciences, School of Medicine, National and Kapodistrian University of Athens, Athens, Greece

3 Laboratory of Anatomy, School of Medicine, University of Crete, Heraklion, Greece

4 Department of Anatomy and Surgical Anatomy, Faculty of Health Sciences, Aristotle University of Thessaloniki, Thessaloniki, Greece

5 Department of Surgical Oncology, St Savvas Cancer Hospital, Athens, Greece 